\title{
Federating Identity Management for a Regional HPC Resource
}

\author{
Jonathon Anderson \\ University of Colorado Boulder \\ Boulder, CO, USA \\ jonathon.anderson@colorado.edu
}

\author{
Thomas Hauser \\ University of Colorado Boulder \\ Boulder, CO, USA \\ thomas.hauser@colorado.edu
}

\begin{abstract}
The University of Colorado Boulder Research Computing group operates RMACC Summit, a shared regional high-performance computing resource managed jointly by the University of Colorado Boulder and Colorado State University and funded by a grant from the National Science Foundation. Access to the resource is additionally available to partner institutions in the Rocky Mountain Advanced Computing Consortium, particularly to institutions without local high-performance computing resources. Users from these institutions would typically be required to acquire an account at the operating institution and then acquire a dedicated account for the resource. Alternatively, a dedicated login environment to differentiate between accounts originating from different institutions could be provided. CU Boulder's Research Computing group has obviated these concerns, providing access to a single, uniform Linux login environment that is used simultaneously by CU Boulder, CSU, and partner institutions facilitated by an XSEDE login service. The use of the existing XSEDE identity and access infrastructure allows CU Boulder's Research Computing group to support a pervasively distributed user community on the RMACC Summit supercomputer without requiring formal identity federation with each RMACC partner institution; and encourages wider familiarity with and adoption of XSEDE in the RMACC user community.
\end{abstract}

\section{CCS CONCEPTS}

- Security and privacy $\rightarrow$ Authentication; Authorization; Distributed systems security; • Information systems $\rightarrow$ Enterprise information systems;

\section{KEYWORDS}

research computing, authentication and authorization, identity federation, XSEDE, AMIE, Duo, sssd

\section{ACM Reference Format:}

Jonathon Anderson and Thomas Hauser. 2018. Federating Identity Management for a Regional HPC Resource. In PEARC '18: Practice and Experience in Advanced Research Computing, fuly 22-26, 2018, Pittsburgh, PA, USA. ACM, New York, NY, USA, 6 pages. https://doi.org/10.1145/3219104.3219113

\section{INTRODUCTION}

The University of Colorado Boulder (CU Boulder) Research Computing (RC) group operates RMACC Summit, a shared regional

Permission to make digital or hard copies of part or all of this work for personal or classroom use is granted without fee provided that copies are not made or distributed for profit or commercial advantage and that copies bear this notice and the full citation on the first page. Copyrights for third-party components of this work must be honored.

For all other uses, contact the owner/author(s).

PEARC '18, fuly 22-26, 2018, Pittsburgh, PA, USA

(C) 2018 Copyright held by the owner/author(s)

ACM ISBN 978-1-4503-6446-1/18/07.

https://doi.org/10.1145/3219104.3219113 high-performance computing resource managed jointly by the University of Colorado Boulder and Colorado State University and funded by a grant from the National Science Foundation. Access to the resource is additionally available to partner institutions in the Rocky Mountain Advanced Computing Consortium (RMACC) [13], particularly institutions without local high-performance computing resources. The RMACC mission is to facilitate widespread effective use of high performance computing throughout the Rocky Mountain region by:

(1) Educating graduate and undergraduate students, faculty, researchers, and industry partners on the use of computational science and high performance computing.

(2) Coordinating multi-institutional efforts to advance research, practice, and education in computational science and to address important regional problems.

(3) Bringing together a broad range of researchers, faculty, and industry partners with a depth of experience and expertise not available at any single institution and facilitate their collaboration in multi-disciplinary and multi-institutional teams.

The RMACC Summit supercomputer is a key component for collaborative and outreach activities of the lead institutions of RMACC to smaller schools in the region.

\section{EXISTING INFRASTRUCTURE}

RMACC Summit is one component of the Research Computing cyberinfrastructure at CU Boulder. This infrastructure also includes a dedicated "Science Network" and "Science DMZ" [4]; a shared high-throughput cluster, "Blanca"; the "PetaLibrary" research data storage system; and training and consulting services. In support of this environment RC operates standard Linux services including an LDAP directory, an account management web portal (RCAMP) [14], and two-factor authentication infrastructure.

Ideally, RC would not operate directory or authentication services at all; but would, instead, use the directory and authentication services provided by the campus Office of Information Technology (OIT). However, limitations in the CU Boulder Active Directory (AD) have prevented us from depending entirely on upstream authentication and authorization services:

- $\mathrm{AD}$ does not provide posixGroups or compatible objects (and existing groups do not provide GIDs).

- Campus policy makes adding external user accounts to AD cumbersome, both intitially and over time.

The RC LDAP directory replicates a subset of existing CU Boulder $\mathrm{AD}$ accounts as $\mathrm{RC}$ accounts (particularly preserving the values of uid and uidNumber); however, it has historically also incorporated RC-internal accounts from NCAR and CU Denver (partners 


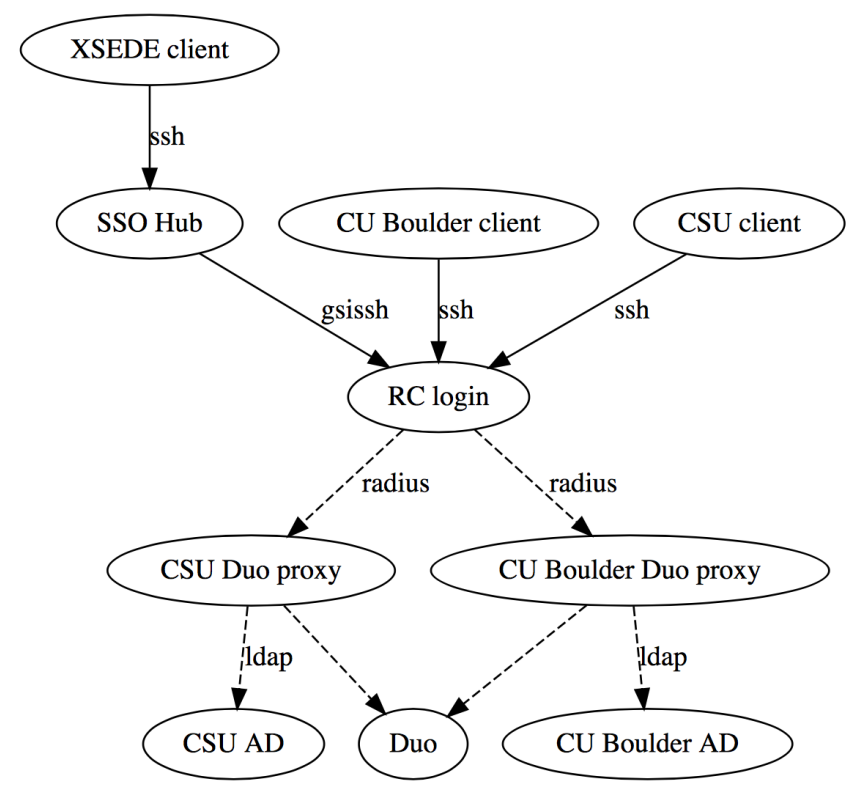

Figure 1: RC authentication components and their interfaces

from previous projects). Because these account names are not defined in upstream AD they have the potential to conflict with new (potentially even existing) upstream accounts. RMACC Summit carries no general entitlement to access for these existing external accounts, giving us the opportunity to deprecate these legacy accounts and refactor the implementation of new external accounts to avoid future namespace collisions.

\section{ACCOUNT MANAGEMENT FOR MULTIPLE COMMUNITIES}

RMACC Summit supports access from three different user communities: CU Boulder, CSU, and RMACC. All three account types are combined at the client using the multiple-domain support of the System Security Services Daemon (SSSD) [17]. External accounts are qualified with domain-specific suffixes to prevent cross-domain name conflicts. Figure 1 provides a general overview of the login path for each account type, and Figure 2 lists the relevant directives from sssd.conf for future reference.

\subsection{CU Boulder Accounts}

Due to the prevalent and continuing use of upstream CU Boulder accounts at RC, we retained these accounts as-is in RC LDAP. Authorized upstream CU Boulder accounts are replicated in RC LDAP with the same uid and uidNumber as in the upstream AD. This replication is handled transparently by RCAMP during an account request process.

Authentication during account request and subsequent login is handled by an instance of the Duo [6] authentication proxy [5] which provides two-factor authentication combining the upstream $\mathrm{AD}$ password and an out-of-band second-factor (e.g., via a mobile application, SMS message, or telephone call). Client applications authenticate to the proxy using the RADIUS protocol [12] which enables access by Linux Pluggable Authentication Modules (PAM) [9] via pam_radius [10]. (Figure 3)

\subsection{CSU Accounts}

CSU accounts are created in much the same way as CU Boulder accounts: an account request is submitted through RCAMP which, in turn, queries the CSU AD to confirm account details (e.g., username and email address). (We do not maintain uidNumber equivalency with CSU AD, instead selecting a uidNumber from a local range.) CSU accounts and groups are created in a discrete OU of the RC $\mathrm{LDAP}^{1}$ to distinguish them from CU Boulder accounts.

By setting use_fully_qualified_names = true in sssd. conf (Figure 2), CSU account and group names are differentiated from CU Boulder names through the use of the suffix @colostate. edu. This suffix guarantees that CSU names do not conflict with current or future CU Boulder names, which are presumed to never have an @colostate. edu suffix.

Separating CSU accounts into a dedicated SSSD domain further supports separating the authentication mechanism for CU Boulder and CSU accounts. Using the proxy_pam_target feature (Figure 2) SSSD can delegate authentication and authorization to a distinct PAM stack for each domain. By coincidence, CSU authentication mirrors CU Boulder and is based on a Duo authentication proxy instance configured to authenticate against the CSU AD and the CSU Duo account; but the support for arbitrary per-domain PAM stacks enables arbitrarily distinct authentication mechanisms to operate within the same environment.

With this configuration in place, CSU users are able to access $\mathrm{RC}$ login services (and all other RC resources) using their existing CSU credentials, only appending the @colostate.edu suffix to their username. Accounts and directories in the RC environment appear as expected, even if the presence of the qualified username is initially surprising. (Figure 4)

\subsection{RMACC Accounts}

The configuration that enables access for CSU accounts works for one external institution, and could theoretically be extended to add an additional domain for each institution for which we would like to provide access; but we observe that the RMACC user community is pervasively distributed, with only a few accounts from any given individual institution. As such, the effort required to coordinate with each institution's identity provider (assuming they were even willing to federate) would be disproportionate to the value gained from the federation. That said, we still did not want to be responsible for assigning usernames, maintaining an internal account namespace authority, or distributing credentials to a significantly geo-distributed user population.

CU Boulder, in agreement with CSU, selected XSEDE [18] as a de facto upstream identity provider for RMACC partner accounts. SSSD configuration is similar to that for CSU accounts, adding an additional xsede. org domain to sssd. conf (Figure 2). Account requests are processed using standard XSEDE processes at the XSEDE user portal ${ }^{2}$ rather than via RCAMP. In contrast with traditional

\footnotetext{
${ }^{1}$ ou $=$ CSU, ou=rc, dc=int, ou=colorado, ou=edu

${ }^{2}$ https://portal.xsede.org
} 


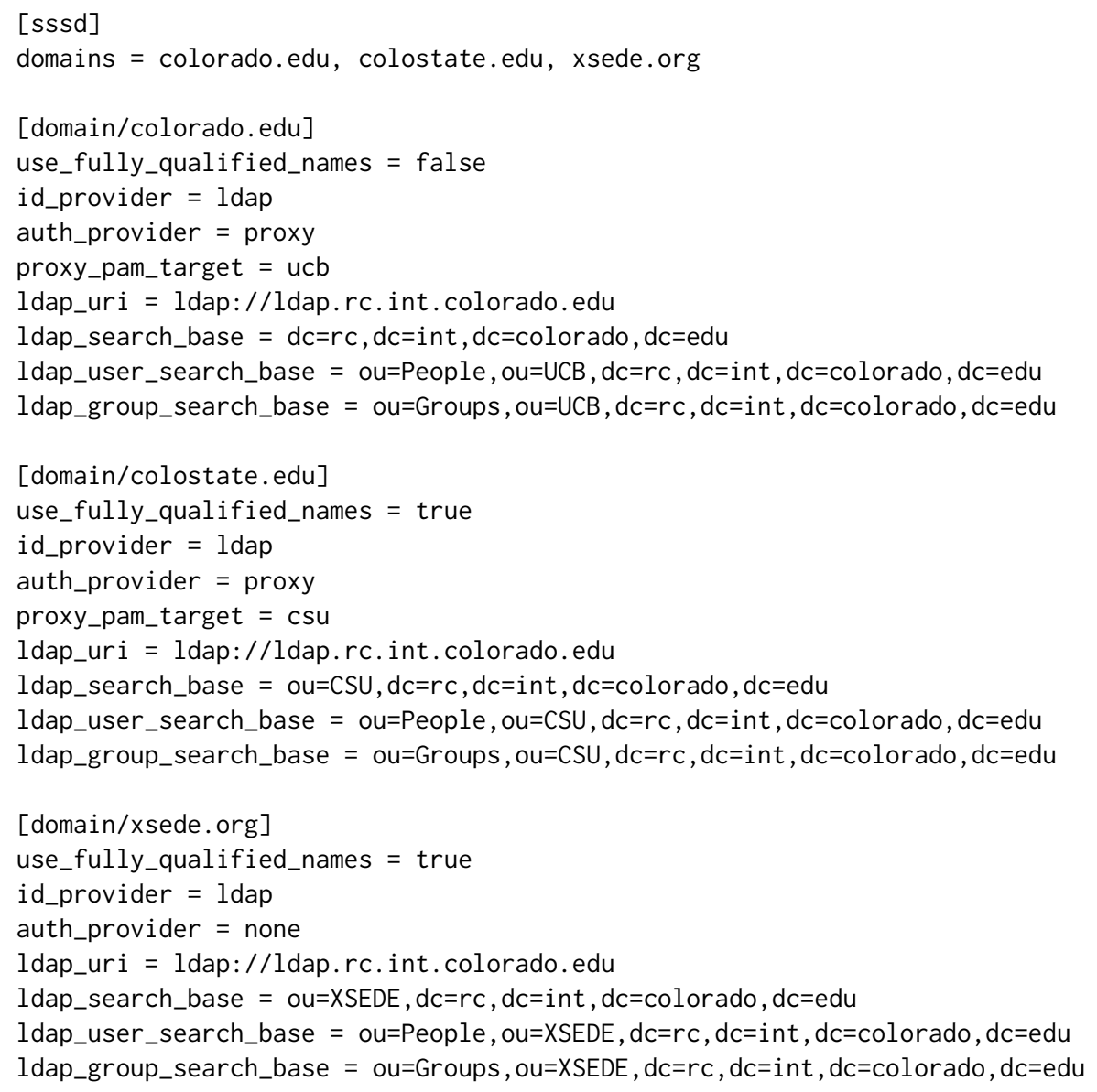

Figure 2: A subset of directives from sssd.conf

localhost: localuser\$ ssh username@login.rc.colorado.edu

[username@login10 ]\$

\section{Figure 3: An example CU Boulder login}

localhost: localuser\$ ssh -1 eid@colostate.edu login.rc.colorado.edu

[eid@colostate. edu@login10 ]\$

\section{Figure 4: An example CSU login}

use of the XSEDE identity management system, RC does not map XSEDE identities to distinct local usernames. Instead we use the XSEDE portal account name as-is, only applying an @xsede.org qualifying suffix. As with CSU accounts, this suffix guarantees that XSEDE names do not conflict with current or future CU Boulder (or CSU) names, which are presumed to never have an @xsede.org suffix.

XSEDE users and service providers authenticate XSEDE accounts using a fork of OpenSSH that supports the Grid Security Infrastructure (GSI) [8] which must be used on both the server and the client This custom client authenticates directly with the XSEDE identity provider, rather than through the the service provider. The identity provider supplies an authentication certificate to the client which is then presented to the service provider for authentication. This negates any need to disclose authentication credentials (i.e., a user password) to the service provider.

\section{THE XSEDE IDENTITY PROVIDER}

The XSEDE account management system AMIE [19] publishes accounts to service providers (SPs) using RabbitMQ [11]. RC uses the reference amie_rabbitmq client application [1] to send and receive AMIE packets as XML documents. Additional glue code [2] was written to discover and parse new incoming AMIE packets, 
localhost: localuser\$ ssh user@login.xsede.org

Please login to this system using your XSEDE username and password:

password:

Duo two-factor login for user

Enter a passcode or select one of the following options:

1. Duo Push to $X X X-X X X-4072$

2. Phone call to $X X X-X X X-4072$

Passcode or option (1-2): 1

Success. Logging you in...

[user@ssohub ] \$ gsissh rmacc-summit

[user@xsede.org@login11 ]\$

Figure 5: An example XSEDE SSO Hub login

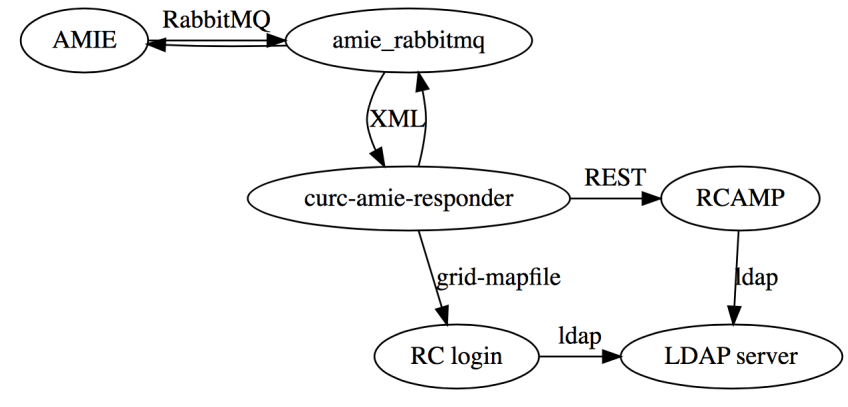

Figure 6: XSEDE account management components and their interfaces

coordinate the account request with RCAMP, generate and respond with appropriate AMIE account creation transaction packets, and maintain grid-mapfile, which enumerates authorized XSEDE accounts (by DN) and maps them to appropriate local accounts (by local username). RCAMP provisions XSEDE accounts and groups in a discrete $\mathrm{OU}$ of the $\mathrm{RC} \mathrm{LDAP}^{3}$ to distinguish them from $\mathrm{CU}$ Boulder and CSU accounts. Figure 6 provides a general overview of the components that provision XSEDE accounts for use in the $\mathrm{RC}$ environment.

\subsection{The XSEDE SSO Hub}

As previously described, GSI authentication for XSEDE accounts requires explicit GSI support in the SSH client; but most RMACC users will not have access to a local installation of a GSI-enabled SSH client. To reduce the authentication burden on end-users, XSEDE has deployed the Single Sign On (SSO) Hub [15], a Linux login server accessible from a traditional SSH client, which authenticates using an XSEDE portal account username, password, and Duo second-factor. This SSO hub is equipped with a local installation of the GSI-enabled OpenSSH clients which can then be used to authenticate to XSEDE service providers. (Figure 5)

The SSO Hub service was extended for use by "level 3" SPs (including RMACC Summit) as part of XCI-36 [3]. The SSO Hub also

\footnotetext{
${ }^{3}$ ou $=X S E D E$, ou $=r c, d c=i n t, o u=c o l o r a d o, ~ o u=e d u$
}

now requires the use of two-factor authentication via an XSEDEprovided Duo instance, fulfilling our local requirement that all computational access be protected by two-factor authentication.

\section{LESSONS LEARNED AND FUTURE IMPROVEMENTS}

\subsection{Namespacing Directories}

Initial attempts to differentiate CSU account directories used subdirectories to provide namespacing. For example, a CSU home directory might have been located at /home/csu/eid/. This was ultimately determined to be more disruptive than the eventual @qualified syntax (e.g., /home/eid@colostate.edu/), as directory namespacing interfered with expected variable substitution templates (e.g., /home/\$USER/). After the initial surprise of seeing qualified usernames, @-qualified directory names were found to be more intuitive for our users and easier to use in documentation.

\subsection{Handling of @ in Perl Scripts}

We have seen a very small number of cases where the presence of the @ character in directory names has caused errors in Perl code due to insufficiently-specific string quoting and the use of a as a sigil for implicit variable interpolation. In cases where fixing the upstream code was impractical, we created directory-namespaced symbolic links (symlinks) for use as aliases for their canonical home directory (e.g., /home/csu/eid -> /home/eid@colostate.edu/). This workaround can also be implemented without administrative intervention by creating symlinks under /tmp/.

\subsection{Cross-domain Group Membership}

We were surprised to discover that groups in one SSSD domain cannot contain users from another domain.

SSSD dynamically presents qualified account names to the Linux name service: they are not stored in LDAP with domain qualifiers. This automatic qualification extends to group names and group member names. As such, an attempt to include ucb_user as a member of csu_group@colostate.edu leads to an invalid member name ucb_user@colostate.edu. Even in the other direction, the addition of csu_user@colostate.edu as a member of 
ucb_group is not honored, despite the lack of any automatic qualification, because SSSD will only relate an account to groups in its own domain, and vice versa.

To work around this limitation, we have, as necessary, created a mirror of relevant groups in collaborating domains, using the same base name and gidNumber. This allows Linux to see users from any domain as members of the same effective group (by gidNumber), even if they are not technically part of the same LDAP group.

This is a suboptimal solution, as the presence of a mirror group in the colorado.edu domain causes files to appear to be owned by it rather than the colostate.edu group. Further development and collaboration may be required with Red Hat to find a better solution long-term; but, so far, this has only been an issue for file system access, which the use of an overlapping gidNumber effectively grants.

\subsection{The Future of GSI Authentication}

The Globus team at the University of Chicago has announced that it would end development of the Globus Toolkit at the end of 2017, including no longer providing security patches by the end of 2018 [16]. Many components of the XSEDE infrastructure are drawn from the Globus Toolkit, including GSI and the GSI-Enabled fork of OpenSSH. In response, XSEDE is coordinating continued support for the Globus Toolkit during its migration to replacement services [20]. XSEDE tentatively plans to authenticate SSH services with OIDC+OAuth2 (via Globus SSH with Globus Auth) but this system is still being developed. Should this implementation be insufficient for the needs of the XSEDE community, GSI-Enabled OpenSSH may continue to be used from the Globus Community Toolkit [7], a community-supported fork of the Globus Toolkit.

\subsection{Globus Authentication}

Somewhat ironically, our use of GSI authentication for XSEDE accounts is incompatible with our existing PAM-based authentication for Globus transfers in our data-transfer environment. Additional investigation and configuration is required to enable XSEDE users to authenticate to our data-transfer nodes.

\subsection{Additional Use of XSEDE Authentication}

Now that XSEDE authentication has been deployed at RC, we may see greater use of XSEDE authenticaiton for external accounts, beyond the RMACC use case. A CU Boulder or CSU user with an existing XSEDE account could conceivably use their XSEDE account to access RC systems; and this pattern makes even more sense if CU Boulder were to deploy a L2 XSEDE resource in the future.

As previously mentioned, legacy NCAR and CU Denver accounts are subject to potential username conflicts due to the lack of any formal namespacing. These accounts, though decommissioned, have been ported to inaccessible SSSD domains to provide necessary namespacing. When an account holder for such a legacy account requires access to an RC system, such accounts have been reprovisioned as an XSEDE-based account.

\section{CONCLUSION}

Through the use of existing software and community infrastructure, Research Computing at the University of Colorado Boulder has assembled a flexible environment capable of supporting users from many institutions at various levels of integration with minimal end-user operational overhead. This enables RMACC Summit to provide compute resources not only to its funding partners but to under-served regional partners. Use of XSEDE infrastructure provides RC with valuable experience, potentially supporting the deployment of future RC services as XSEDE services; and also encourages familiarity with and adoption of XSEDE resources by the RMACC community, supporting future use of other XSEDE resources.

\section{ACKNOWLEDGMENTS}

The authors would like to thank Rob Light at the Pittsburgh Supercomputing Center and JP Navarro at Argonne National Laboratory for helping us at CU Boulder Research Computing in our first integration with XSEDE systems and practices.

This work is in support of RMACC Summit, itself supported by the National Science Foundation under grants numbered ACI1532235 and ACI-1532236.

This work used the Extreme Science and Engineering Discovery Environment (XSEDE), which is supported by National Science Foundationrant number ACI-1548562, specifically as the resource rmacc-summit. colorado.xsede.org at the University of Colorado Boulder through allocation TG-CDA170006, "University of Colorado XSEDE Campus SSO Integration."

\section{REFERENCES}

[1] amie_rabbitmq 2018. Reference implementation of a RabbitMQ client for the XSEDE AMIE system. (2018). https://github.com/ResearchComputing/amie_ rabbitmq Accessed: 2018-03-27.

[2] Jonathon Anderson. 2018. (2018). https://github.com/ResearchComputing/ curc-amie-responder Accessed: 2018-03-27.

[3] Jim Basney. 2018. Enable L3 resource logins via XSEDE using login allocations. (2018). https://software.xsede.org/display/XCI-36 Accessed: 2018-03-27.

[4] Eli Dart, Lauren Rotman, Brian Tierney, Mary Hester, and Jason Zurawski. 2013. The Science DMZ: A Network Design Pattern for Data-intensive Science. In Proceedings of the International Conference on High Performance Computing, Networking, Storage and Analysis (SC '13). ACM, New York, NY, USA, Article 85, 10 pages. https://doi.org/10.1145/2503210.2503245

[5] Duo Authentication Proxy 2018. Duo Authentication Proxy. (2018). https: //duo.com/docs/authproxy_reference Accessed: 2018-03-27.

[6] Duo Security, Inc. 2018. Duo Security, Inc. (2018). https://duo.com/about Accessed: 2018-03-27.

[7] Grid Community Forum 2018. Community-based support for core software packages in grid computing. (2018). https://gridcf.org Accessed: 2018-03-27.

[8] GSI-SSH 2018. GSI-Enabled OpenSSH. (2018). http://grid.ncsa.illinois.edu/ssh/ Accessed: 2018-03-27.

[9] Linux PAM [n. d.]. Linux-PAM. ([n. d.]). http://www.linux-pam.org Accessed: 2018-05-30.

[10] pam radius [n. d.]. FreeRADIUS pam_radius. ([n. d.]). https://github.com/ FreeRADIUS/pam_radius Accessed: 2018-05-30.

[11] RabbitMQ 2018. RabbitMQ: an open source message broker. (2018). https: //www.rabbitmq.com Accessed: 2018-03-27.

[12] Carl Rigney, Allan C. Rubens, William Allen Simpson, and Steve Willens. 2000. Remote Authentication Dial In User Service (RADIUS). (June 2000). https: //tools.ietf.org/html/rfc2865

[13] Rocky Mountain Advanced Computing Consortium, The 2018. The Rocky Mountain Advanced Computing Consortium. (2018). https://rmacc.org/about Accessed: 2018-03-27.

[14] Zeb Sampedro. 2018. (2018). https://github.com/ResearchComputing/RCAMP Accessed: 2018-03-27.

[15] sso hub [n. d.]. XSEDE Single Sign On Hub. ([n. d.]). https://portal.xsede.org/ single-sign-on-hub 
[16] Support for open source Globus Toolkit will end as of January 2018 2017. Support for open source Globus Toolkit will end as of January 2018; The Globus cloud service and Globus Connect are unaffected. (May 2017). https://github.com/globus/ globus-toolkit/blob/globus 6 branch/support-changes.md Accessed: 2018-03-27.

[17] System Security Services Daemon 2018. System Security Services Daemon (SSSD) (2018). https://fedoraproject.org/wiki/Features/SSSD Accessed: 2018-03-27.

[18] J. Towns, T. Cockerill, M. Dahan, I. Foster, K. Gaither, A. Grimshaw, V. Hazlewood, S. Lathrop, D. Lifka, G. D. Peterson, R. Roskies, J. R. Scott, and N. WilkinsDiehr. 2014. XSEDE: Accelerating Scientific Discovery. Computing in Science \& Engineering 16, 5 (Sept.-Oct. 2014), 62-74. https://doi.org/10.1109/MCSE.2014.80

[19] XSEDE AMIE 2018. XSEDE local account and accounting transaction transport. (2018). https://software.xsede.org/global-software-and-service-component/amie Accessed: 2018-03-27.

[20] XSEDE Response to the Globus Toolkit end-of-support announcement 2018. XSEDE response to the Globus Toolkit end-of-support announcement. (March 2018). https://software.xsede.org/news/ xsede-response-globus-toolkit-end-support-announcement Accessed 2018-03-27. 